\title{
Analysis of Automated Aircraft Conflict Resolution and Weather Avoidance
}

\author{
John F. Love ${ }^{*}$ and William N. Chan. ${ }^{\dagger}$ \\ NASA Ames Research Center, Moffett Field, CA, 94035 \\ Chu Han Lee \\ University of California Santa Cruz, Moffett Field, CA, 94035
}

\begin{abstract}
This paper describes an integrated solution to traffic conflict detection and resolution with weather avoidance in the Center-TRACON Automation System. An automatic conflict resolution algorithm developed and tested to resolve aircraft-to-aircraft conflicts is modified to reroute aircraft around convective weather constraints while avoiding traffic conflicts. The approach extends algorithms which resolve aircraft-to-aircraft conflicts to also resolve aircraft-to-forecast weather conflicts. Alternatively, a separate algorithm that solves aircraft to weather conflicts would have to be integrated with the current method to solve both aircraft and weather conflicts. This evaluation limited conflict resolutions to horizontal maneuvers due to software limitations in the research software that are being addressed. Simulation results show 96 percent of aircraft-to-aircraft conflicts without any weather constraints were successfully resolved. In high traffic scenarios with moderate weather, 91 percent of conflicts were resolved, while in high traffic with bad weather 72 percent of conflicts were resolved.
\end{abstract}

\section{Introduction}

$\mathrm{I}_{\mathrm{c}}^{\mathrm{N}}$ $\mathrm{N}$ order for Air Traffic Management Decision Support Tools based on real-time trajectory automation, such as the Center-TRACON Automation System, to work in all weather conditions, convective weather information must be integrated into aircraft traffic conflict detection and resolution automation. To address improving the usefulness of the Center-TRACON Automation System in all weather conditions, this paper discusses the development of an algorithm to automatically route aircraft around weather constraints while keeping them from conflicting with other aircraft. Currently, the Center-TRACON Automation System does not account for convective weather in its tools such as the Traffic Management Advisor ${ }^{1}$, Direct-To ${ }^{2}$ and in its conflict probe functionality. The FAA deployed the Traffic Management Advisory across the country and the FAA's Research, Engineering and Development Advisory Committee recommends "...enhancing the Traffic Management Advisor to achieve a weather sensitive arrival planning tool." ${ }^{3}$

This study evaluates the extension of an algorithm to automatically resolve aircraft to aircraft conflicts, already in the Center-TRACON Automation System, to also avoid weather conflicts. The approach described in this paper was taken for the following reasons. Using a separate weather avoidance algorithm would require that it work with the current aircraft conflict resolution algorithm in the Center-TRACON Automation System or an entirely new algorithm that resolved both weather and aircraft conflicts be created. Another reason is to utilize the aircraft resolution logic that was designed to create resolutions typical of the ones used by controllers as those resolutions may be acceptable to solve weather and aircraft conflicts. This work will also evaluate the efficacy of this simple extension to serve as a baseline of performance for other such algorithms implemented in the Center-TRACON Automation System. The algorithm that was extended is based on auto-resolver logic that was developed by Erzberger $^{4}$ and used to evaluate weather avoidance logic without mitigating aircraft conflicts in the Airspace Concept Evaluation System. ${ }^{5}$ Weather constraints for this study were defined by the Convective Weather Avoidance Model $^{6}$ which was integrated in the Center-TRACON Automation System. This weather model

\footnotetext{
* Aerospace Engineer, Systems Modeling and Optimization Branch, Moffett Field MS 210-8, Member.

${ }^{\dagger}$ Chief, Aerospace High Density Operations Branch, Moffett Field MS 210-8, Senior Member.

* Senior Programmer/Analyst, University of California Santa Cruz/University Affiliated Research Center, Moffett Field MS 210-8.
} 
translates convective weather information to identify convective regions of airspace that pilots are likely to deviate around.

This paper describes how the resolution algorithm was extended and the Convective Weather Avoidance Model used for this study. A methodology section describes how the Center-TRACON Automation System was modified to detect and resolve weather conflicts. Simulation results discuss the effectiveness of this algorithm to solve both aircraft and weather conflicts. Finally, how the resolutions affect flight times for the simulation scenarios are discussed. The paper ends with conclusions and suggestions for future work to improve tactical aircraft routing considering weather constraints.

\section{Simulation Methodology}

The automatic aircraft-to-aircraft conflict detection and resolution module in Center-TRACON Automation Software (CTAS) was modified to detect and resolve predicted aircraft encounters with weather. Regions of en route airspace with convective weather that aircraft were likely to avoid were constraints. Weather constraints were defined by the Convective Weather Avoidance Model (CWAM). Aircraft encounters with weather are treated the same as aircraft conflicts with other aircraft.

\section{A. Center-TRACON Automation System Software Baseline}

CTAS is a suite of integrated decision support tools that uses 4-D trajectory modeling and analysis to provide information and advisories to air traffic controllers. These advisories include conflict detection and resolution, direct-to routings with one minute or more in savings, and airport arrival rate scheduling. The FAA version of the Traffic Management Advisor (TMA), ${ }^{7}$ one element of CTAS, has been deployed by the FAA to all 20 en route control centers but it lacks any ability to automatically detect and mitigate impacts to its computed schedule based on convective weather constraints. The FAA version differs from the NASA research version used in this study but their basic functionality remains the same. CTAS employs radar track and flight plan data from the FAA host computer, wind data from the National Weather Service, and a database of aircraft type performance models. These data allows the Trajectory Synthesizer (TS) module to integrate the equations-of-motion for each aircraft type to create a 4-D trajectory. The 4-D trajectories are tested for both conflicts with other aircraft as well as intersection with weather constraints that should be avoided. In this study, the aircraft automatically fly the 4-D trajectories that were computed to be both conflict free of aircraft and weather.

\section{B. The Convective Weather Avoidance Model}

The Convective Weather Avoidance Model (CWAM) developed by MIT/Lincoln Laboratory is described in DeLaura and Evans. ${ }^{8}$ CWAM is a statistically based model that translates convective weather information to probabilities the pilot will deviate around weather. CWAM is based on the analysis of approximately 500 en route flight trajectories through a single en route center from five different days in 2003 with significant convective weather. DeLaura and Evans found that the difference between flight altitude and the radar echo top was the most accurate predictor of aircraft deviation around convective weather. The vertical integrated liquid (VIL), which measures precipitation intensity, played a secondary role. The Corridor Integrated Weather System (CIWS), ${ }^{9}$ also developed by MIT/Lincoln Laboratory, is composed of echo tops and VIL data from weather radars and it provides 2-hour convective forecasts updated every 5 minutes with 5-minute forecast time-steps. The CIWS data are processed by CWAM to create the probabilistic weather avoidance polygons that are a function of echo top height and VIL intensity at each flight level from FL250 to FL450 ft. CWAM also creates forecast polygons based on the CIWS forecast. Since CWAM has forecasts out to 2-hours, the predicted 4-D aircraft trajectories are timesynchronized with the CWAM polygons when checking for conflicts. Time-synchronization is defined as comparing the time step in the 4-D aircraft trajectory against the closet time-step of the CWAM polygon.

The avoidance logic is the same for the aircraft-to-aircraft resolutions with the additional constraint of having to avoid CWAM polygons of 80 percent deviation probability. These probabilities were shown in a previous study to be a reasonable surrogate for determining areas pilots would avoid flying through. ${ }^{8}$ Clearly, other deviation probabilities can be used as Grabbe, et al., ${ }^{10}$ found good results for their study of weather avoidance without considering aircraft conflicts using 60 percent CWAM deviation probabilities.

\section{Aircraft Conflict Resolution Algorithm}

An automatic conflict resolution algorithm described by Erzberger ${ }^{11}$ that resolves aircraft-to-aircraft conflicts has been integrated into CTAS. The algorithm incorporates procedures and heuristics that are typically used by air traffic controllers to resolve conflicts in today's operations. Aircraft conflicts are resolved one pair at a time using 
altitude amendments, horizontal route amendments, and speed changes. The algorithm resolves all combinations of conflicts between cruising, descending, and climbing aircraft. Conflict resolution maneuvers are designed to minimize change in flying time delay relative to the original flight trajectory. The automatic conflict resolution algorithm first checks a direct route to a downstream fix on an aircraft route of flight. These so called "Direct-To" routes can often resolve traffic conflicts, save flying time for airspace users, and are operationally acceptable. ${ }^{2}$ If a direct route does not resolve the conflict, the algorithm prioritizes the heuristic maneuvers for the problem being solved and trial plans each maneuver to check for secondary aircraft conflicts until a resolution is found. The separation criteria for avoiding other en route aircraft were $1000 \mathrm{ft}$ vertical and 5 nautical miles horizontal.

\section{Applying the Resolution Algorithm to Weather Conflicts}

As a first approximation to an automated system of resolving both aircraft-to-aircraft and aircraft-to-weather conflicts, the Advanced Airspace Concept resolution algorithm in CTAS was modified to treat trajectories crossing into a CWAM polygon as head on aircraft conflicts. This method was chosen to explore if this simple extension could be used to solve both aircraft and weather conflicts. If this algorithm cannot be extended, integrating a separate weather avoidance algorithm would require that it work with the current aircraft to aircraft conflict resolver or create a new aircraft and weather conflict resolution algorithm. This implementation abstracted a weather conflict as a head-on conflict with the CWAM polygon because it does not favor either a right or left turn solution. The data sent to the resolution algorithm is based on resolving a conflict at the point where the trajectory intersects the CWAM polygon.

Only horizontal resolutions were used in this study. Altitude maneuvers were not used in this study for weather avoidance because it was found that the version of CTAS used in this study would temporarily assign a lower altitude sending aircraft under the CWAM polygons, which is not an acceptable solution. This limitation is currently being addressed.

Figure 1 shows a flowchart for the iterative resolution process investigated for this study. There are two detection modules, one for aircraft conflicts and another for weather. The CTAS en route module processes the flight plan data and passes the position and velocity states to the TS to create the 4-D trajectory. The module then passes the trajectory information to two parallel aircraft and weather detection modules. The aircraft detection module looks for aircraft-to-aircraft conflicts predicted to occur within the next 20 minutes. The weather detection module compares the aircraft's next 20-minute trajectory with the forecast CWAM polygons.

The look-ahead time depends on the trajectory uncertainty, which is too large for conflict predictions beyond 20 minutes. CTAS' software architecture is designed to function in a real-time radar tracking environment. CTAS normally makes trajectory predictions based on the aircraft type and performance data. There can be differences between radar track and calculated trajectories due to CTAS not having all the required information, such as the weight of the aircraft. The difference between the actual and predicted aircraft locations results in uncertainty, which grows with time. Restricting look-ahead times to 20 minutes is based on current practice to keep the uncertainty within operational limits. This study uses recorded aircraft tracks but they are input to CTAS as if they originated from an FAA radar host. In this simulation, the aircraft positions are determined by their recorded radar tracks below 10,000 ft. Above this altitude, the flight states are determined by the predicted trajectory information and there is no more uncertainty with the aircraft's trajectory but other climbing and descending aircraft trajectories may still depend on the recorded data.

If a conflict is detected, each detection module prioritizes the list of conflicts based on time to conflict and chooses which to send on to the resolution algorithm module. Because of assumed trajectory uncertainty, the detection module queues aircraft-to-aircraft conflicts on the list that are not predicted to occur within 8 minutes. The aircraft-to-weather conflicts are queued until predicted to occur within 12 minutes. The weather conflicts were given more time to reduce the turning angle required for resolution. Weather systems can be much larger than the 5 nautical mile separation criteria that the resolution algorithm was designed to avoid. Successful resolutions were required to be clear of weather polygons but not by any specific distance. Assuming modern aircraft travel at 8 nautical miles per minute, beginning the search for resolutions at the 12-minute point means the algorithm begins attempting to resolve the weather conflicts about 96 miles from the conflict.

The resolution algorithm applies the heuristics for conflict resolutions based on the particular problem. In the case of an encounter with weather, the head on conflict geometry is passed to the algorithm. When a resolution was generated, the algorithm checks for secondary conflicts with other aircraft or CWAM polygons. If no secondary conflicts were found, the aircraft flight plan was amended, and the algorithm moves onto the next conflict received. If no resolution was found in the current 12-second time cycle, the conflict was resent in the next time cycle. This process continues until the time to conflict was below specified time limits. The limits were 3 minutes for aircraftto-aircraft conflicts and 1 minute for aircraft-to-weather conflicts. For conflicts involving weather, the algorithm 
had the additional constraint that it could only change the path of the aircraft. This may have the side effect of decreasing the success rate in congested airspace when reroutes cause secondary conflicts.

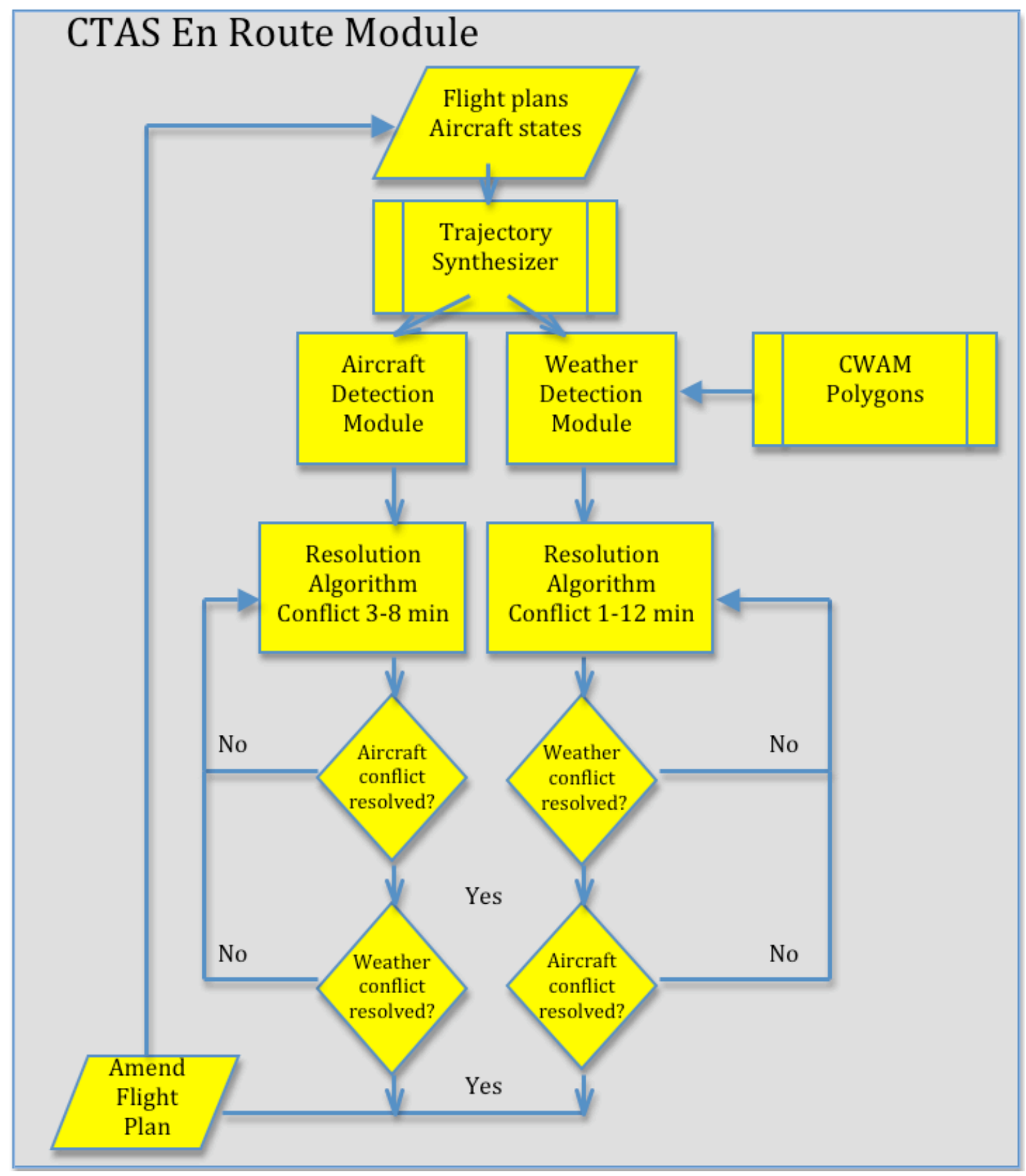

Figure 1. Flowchart of aircraft and weather conflict resolution process 


\section{E. Automatic Aircraft and Weather Resolution}

Figures 2 through 5 show an example of an integrated aircraft/weather conflict resolution for a Northeast bound aircraft at FL350. Shown in all the figures are the CTAS flight data block for the maneuvered aircraft, the flight plan route, the high altitude sector boundaries in Indianapolis Center airspace, the CWAM weather polygons, and other traffic. Figure 2 depicts the original flight plan trajectory. Other aircraft in the region appear as diamond and triangle symbols, though not all are at the same altitude. The altitude dependent weather polygons are created by CWAM representing an 80 percent probability of deviation. The intersection of the trajectory with the CWAM polygon shown in Fig. 2 is more than 20 minutes away so it is not a detected weather conflict. Figure 3 shows the aircraft at the same location and time but superimposed with the forecasted CWAM polygon 15 minutes in the

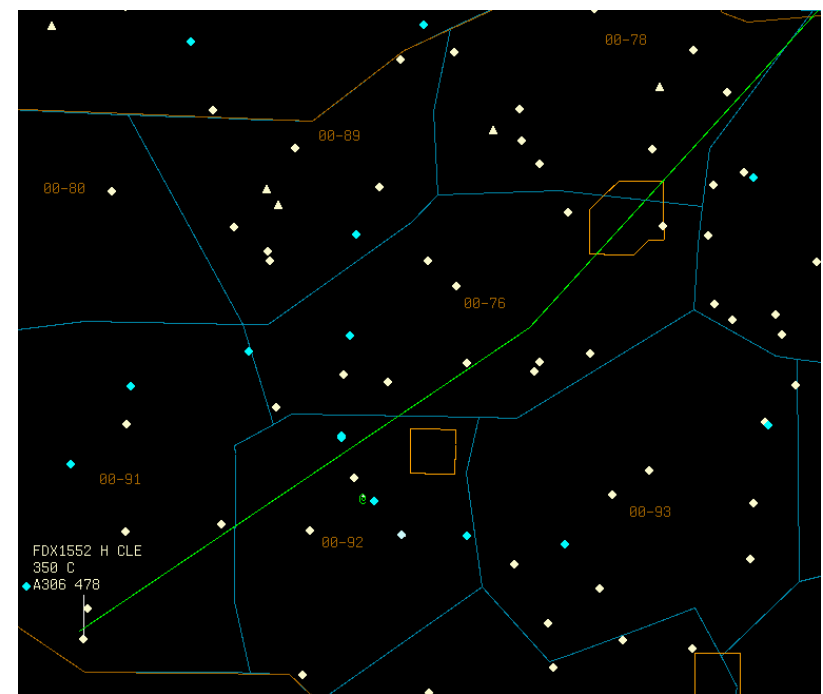

Figure 2. Example showing current aircraft position and weather at time 0 .

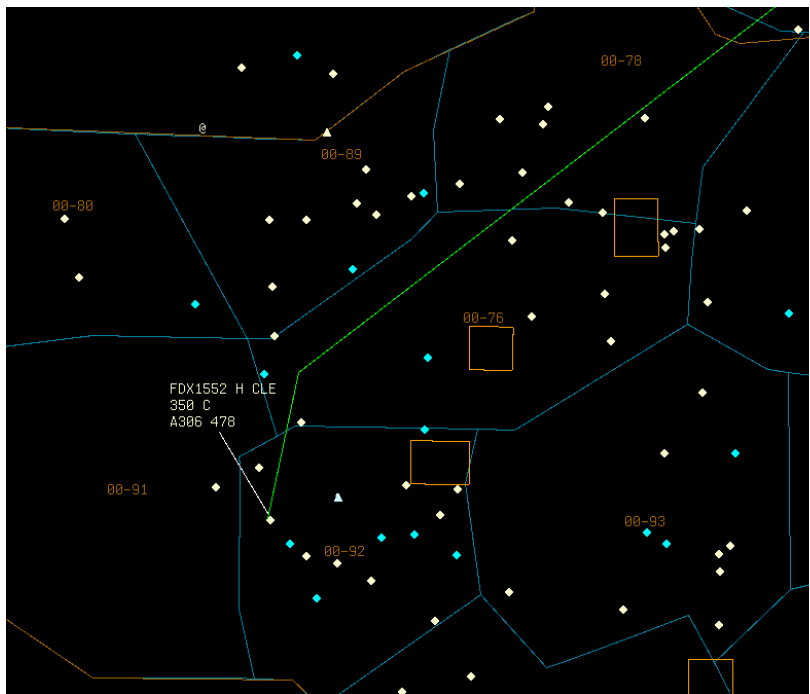

Figure 4. Updated aircraft position and weather at time +5 minutes.

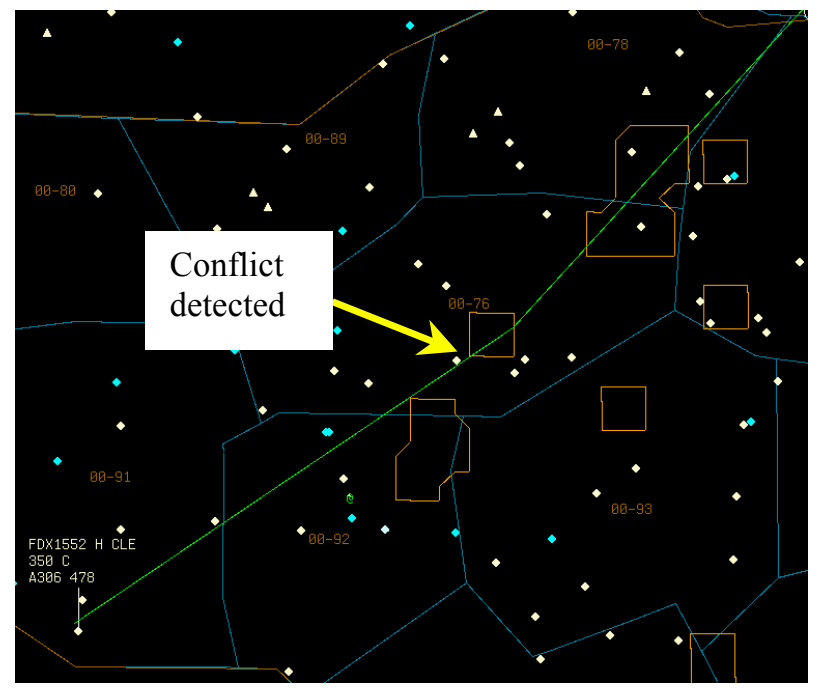

Figure 3. Example showing current aircraft position at time 0 and forecast weather conflict at time $t+15$.

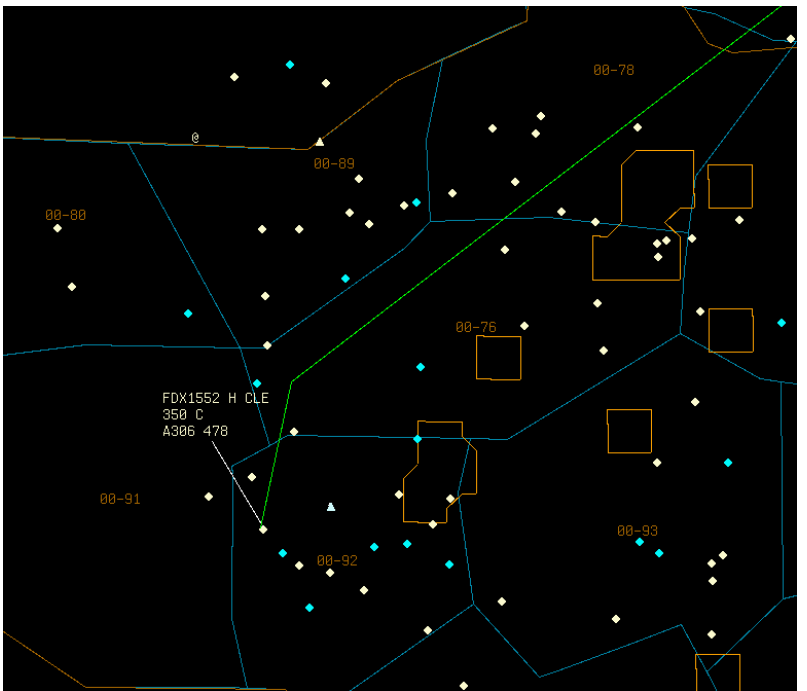

Figure 5. Aircraft's position at time +5 and forecast weather conflict at time $+\mathbf{1 5}$ minutes.

future. The arrow points to the approximate location of the detected aircraft-to-weather conflict. By comparing Figs. 2 and 3, the relative locations of the current and forecast weather polygons can be deduced by noting the location of the sector boundary. The intersection of the aircraft trajectory and CWAM polygon in Fig. 2 is farther 
away than in Fig. 3. The forecast conflict allows more time to detect and resolve weather conflicts rather than waiting for an update to the weather at a future time.

Figures 4 and 5 illustrate the weather avoidance algorithm's conflict free flight plan amendment. Figure 4 shows the same aircraft at time plus 5 minutes. The aircraft is predicted to intercept the CWAM polygon in 10 minutes. At this time, the resolution algorithm has found an acceptable solution free of secondary traffic and weather conflicts. The aircraft's flight plan is amended and the trajectory synthesizer module calculates a new 4-D trajectory, which is displayed in Figure 4. Figure 5 is another view of the aircraft location at time plus 5 minutes with new flight plan superimposed with the forecast weather the original trajectory would have intersected at time plus 15 minutes. This is the weather conflict detected and sent to the resolution algorithm, which was resolved.

\section{Simulation}

For this study, 5.5 hours of high altitude traffic data from December 21, 2007 recorded in Indianapolis Center (ZID) were split into two traffic levels (medium and high) and combined with three different weather scenarios. Indianapolis Center was selected because the required weather data were available for this region. In the simulation, aircraft would fly their original flight plan according to the radar track data and computed 4-D trajectories and rerouted if a weather or aircraft conflict occurred. Data for all six runs were collected and compared. Total aggregate airborne delay times in ZID center were also archived for evaluating the effect of rerouting.

\section{A. Traffic Scenarios}

The traffic and weather scenarios were chosen from different days so the traffic input to the simulation would not be influenced by actual weather deviations. The date of the traffic data was chosen as Friday, December 21, 2007, from 0600 to 1130 Eastern Standard Time. This was a busy day at the beginning of the holiday travel season with clear weather.

Figure 6 shows the recorded aircraft count for that date between the hours of 0600-1130 EST. This traffic file provided both the medium and high traffic scenarios as the aircraft counts varied across this time period. The two traffic level bands are annotated in Fig. 6. The medium traffic scenario was selected as the period from 0600 EST to 0830 EST, the high traffic scenario was selected as the period from 0845 EST to 1115 EST. The first 15 minutes of the recorded data were removed after the simulation to eliminate cases where aircraft either initially start out inside a CWAM polygon or the aircraft is already in conflict and there is insufficient time to find a resolution. For each scenario, only 2 hours of data were analyzed. The medium traffic scenario started with around 100 aircraft and increased to about 200 aircraft and the high traffic scenario started with over 250 and increased to about 340 aircraft.

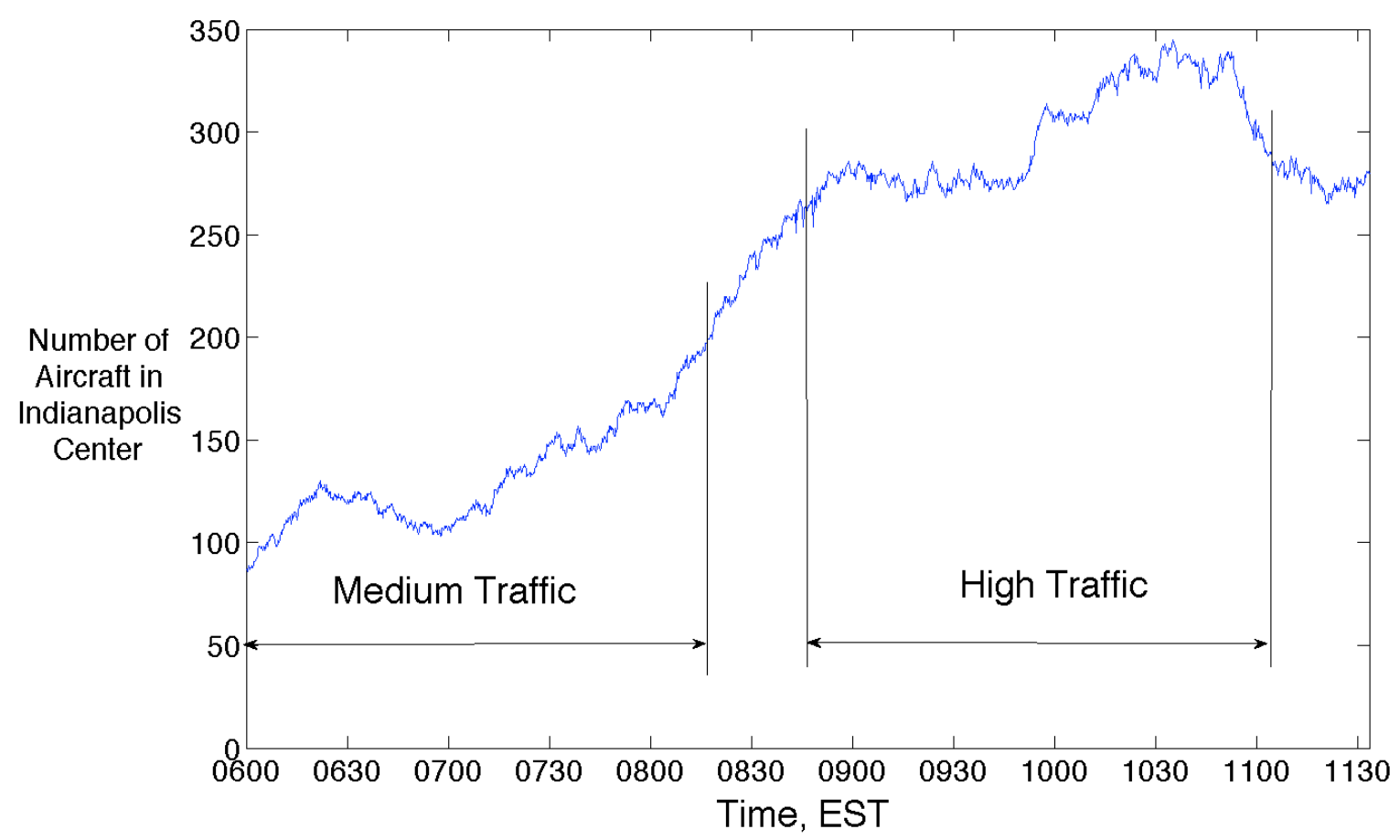

Figure 6. Aircraft count versus time recorded in Indianapolis Center on December 21, 2007 


\section{B. Weather Scenario}

Weather data were chosen based on the amount of convective weather coverage in ZID by inspection of NEXRAD National Reflectivity Mosaics from the National Climatic Data Center. Based on these criteria, June 19, 2007 (bad), July $10^{\text {th }}, 2007$ (moderate), and July $24^{\text {th }}, 2007$ (none) were selected for the three weather scenarios. The terms bad, moderate, and none refer to the overall area coverage.

Figures 7 and 8 show the radar reflectivity data from the bad and moderate scenario, respectively, within ZID airspace. ${ }^{12}$ The white square shown in the figure is the approximate boundary of ZID airspace. According to FAA Operational Network database, the delays on these days were predominately due to weather and not other factors such as equipment problems, high volume of aircraft, runway closures, or other issues.

No wind data were included in the simulation. This study focused on resolutions so winds, though not added, will affect the resolution results but does not change the fact this algorithm can solve aircraft and weather conflicts.

The CIWS radar data consists of an observation at the current time and a two-hour forecast in 5-minute increments. Although these data are updated every 5 minutes, a processing time lag was accounted for in the simulation so new weather data were limited to every 15 minutes. Therefore, each two-hour simulation included 8 CWAM weather updates, one every 15 minutes.

\section{Results}

The overall results for all six scenarios are presented first. Then, a detailed look at the success rates of aircraft-to-aircraft and aircraft-to-weather conflicts along with the distribution of maneuvers is presented. Next, the impacts of aircraft reroutes are presented in terms of time savings and delays. Finally, an example is used to illustrate the real-time schedule impact of the weather rerouting.

\section{A. Overall Results}

Figure 9 shows the overall success rates of the six traffic/weather scenarios investigated in this study. There was no uncertainty due to forecast prediction accuracy or aircraft trajectory uncertainty. The

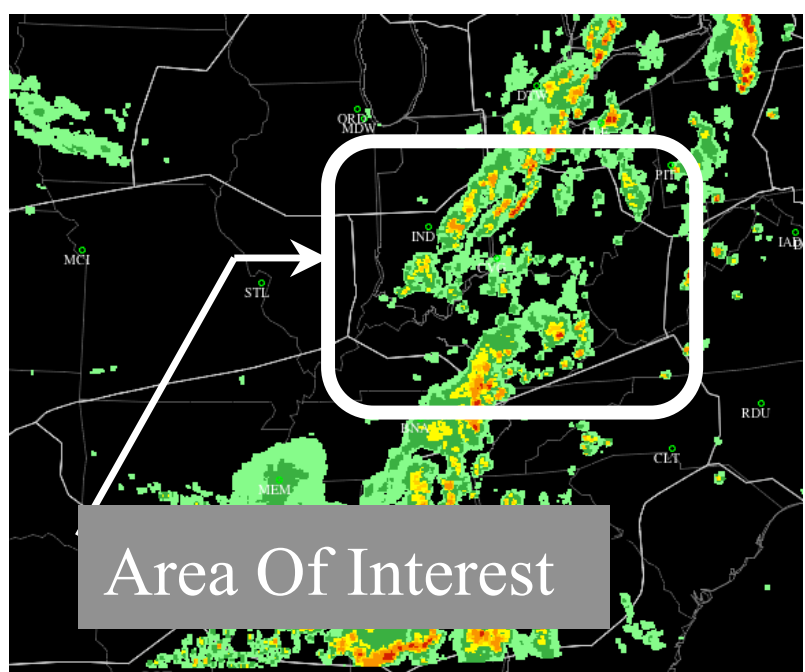

Figure 7. June 19, 2007 2100Z radar reflectivity image on a "bad weather day"

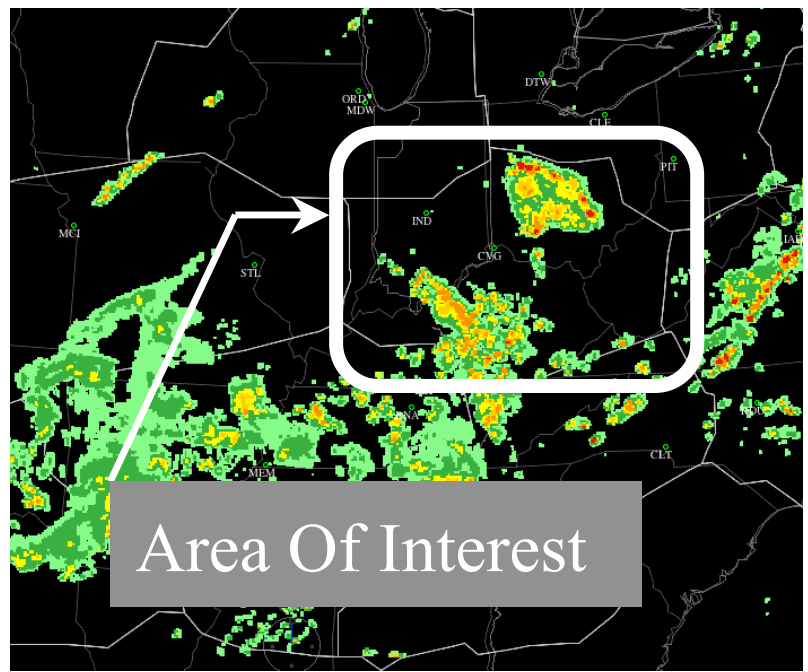

Figure 8. July 10, 2007 1700Z NEXRAD radar reflectivity image on a "moderate weather day" aircraft automatically flew their conflict resolution advisories as proposed by the resolver. Success means that a conflict resolution trajectory that resolves the detected traffic or weather conflict and does not create any other traffic and weather conflict was found. The overall success rates, shown in green, include both aircraft-to-aircraft and aircraft-to-weather conflicts. The success rates for aircraft-to-aircraft conflict resolutions without secondary aircraft or weather conflicts remain above 96 percent for all traffic and weather scenarios. In the unresolved high traffic, no weather, aircraft-to-aircraft conflicts, all eight were descending aircraft that had secondary conflicts. The algorithm was able to resolve at least 91 percent of the conflicts under the moderate weather scenario for either traffic levels. The bad weather scenario dropped the success rates down to about 72 percent. 


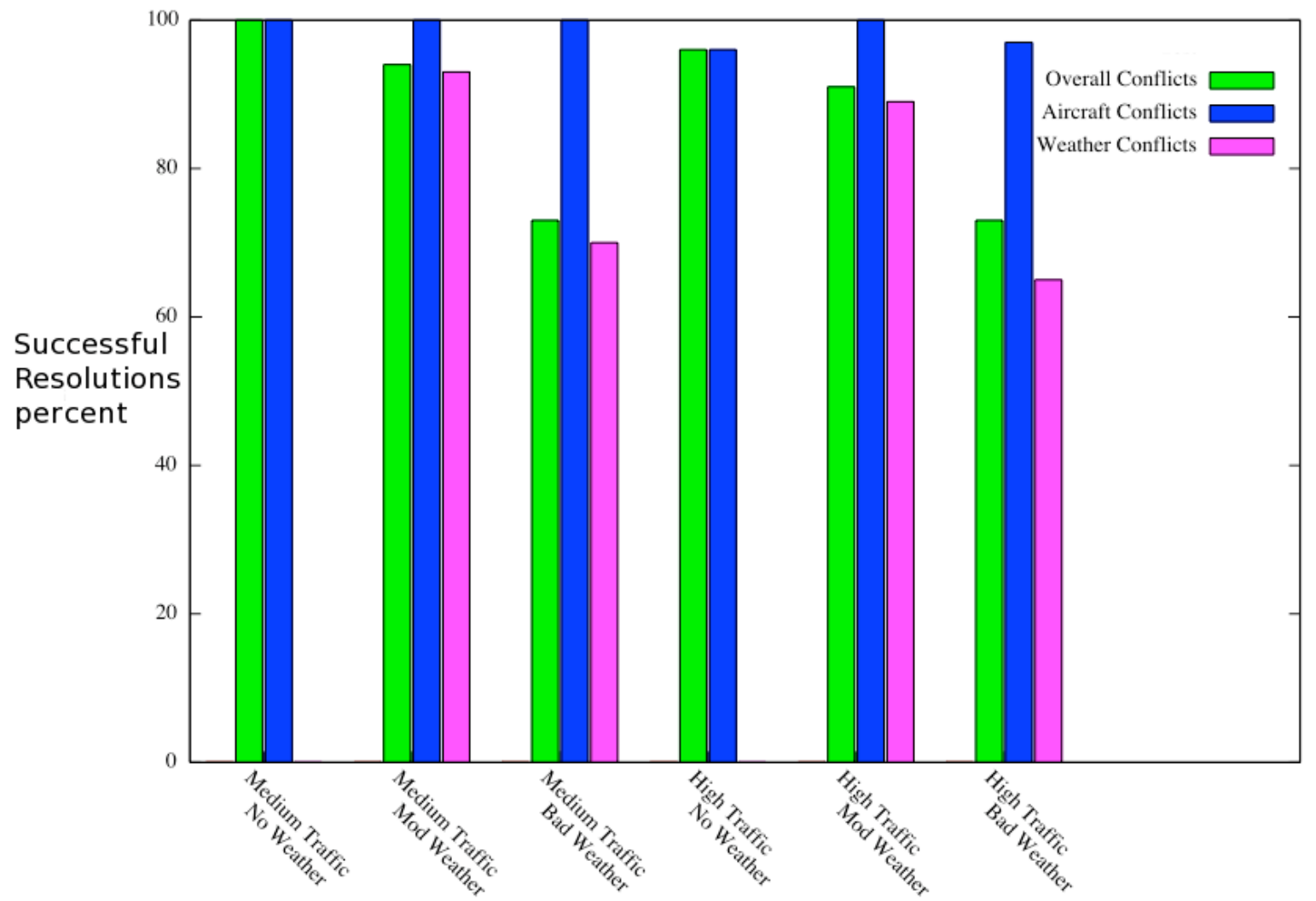

Figure 9. Simulation results for the traffic and weather scenarios

Table 1 shows the total number of conflicts detected during each scenario and corresponding success rate. For the medium traffic and moderate weather scenario the total number of conflicts increased by 214 percent or more than 3 times the no weather scenario. The bad weather scenario had more than 6 times as many aircraft and weather conflicts as the no weather baseline. For the high traffic level, the numbers of moderate and bad weather conflicts were 2 and 4 times the baseline, respectively.

Table 1. Individual and Combined Aircraft and Weather Conflict Resolution Success Rates

\begin{tabular}{ll|ll|l|ll|l|l|l}
\hline \hline $\begin{array}{l}\text { Traffic } \\
\text { level }\end{array}$ & $\begin{array}{l}\text { Weather } \\
\text { level }\end{array}$ & $\begin{array}{l}\text { Number } \\
\text { of } \\
\text { aircraft } \\
\text { conflicts } \\
\text { resolved }\end{array}$ & $\begin{array}{l}\text { Aircraft } \\
\text { conflicts } \\
\text { not } \\
\text { resolved }\end{array}$ & $\begin{array}{l}\text { Aircraft } \\
\text { success, } \\
\text { percent }\end{array}$ & $\begin{array}{l}\text { Number } \\
\text { of } \\
\text { weather } \\
\text { conflicts } \\
\text { resolved }\end{array}$ & $\begin{array}{l}\text { Weather } \\
\text { conflicts } \\
\text { not } \\
\text { resolved }\end{array}$ & $\begin{array}{l}\text { Weather } \\
\text { success, } \\
\text { percent }\end{array}$ & $\begin{array}{l}\text { Total } \\
\text { number } \\
\text { of } \\
\text { conflicts }\end{array}$ & $\begin{array}{l}\text { Total } \\
\text { success, } \\
\text { percent }\end{array}$ \\
\hline \hline \multirow{3}{*}{ Med. } & None & 96 & 0 & $100 \%$ & - & - & - & 96 & $100 \%$ \\
& Moderate & 62 & 0 & $100 \%$ & 220 & 17 & $93 \%$ & 299 & $94 \%$ \\
\hline \hline & Bad & 69 & 0 & $100 \%$ & 375 & 162 & $70 \%$ & 606 & $73 \%$ \\
\hline \multirow{3}{*}{ High } & None & 219 & 8 & $96 \%$ & - & - & - & 227 & $96 \%$ \\
& Moderate & 62 & 0 & $100 \%$ & 335 & 38 & $89 \%$ & 435 & $91 \%$ \\
& Bad & 197 & 7 & $97 \%$ & 492 & 263 & $65 \%$ & 959 & $72 \%$ \\
\hline \hline
\end{tabular}

Table 1 separates the aircraft-to-aircraft and aircraft-to-weather conflict resolution success rates. Notice that the number of aircraft-to-aircraft conflicts decreases with the addition of moderate weather and increases for the bad weather. Inspection of the resolutions revealed the weather rerouting eliminates some aircraft-to-aircraft conflicts. 
The overall success rate decreases with increasing weather level because the algorithm can only change the flight path of the aircraft. In an aircraft-to-aircraft conflict, the resolution algorithm can alter the flight path of either aircraft. In congested airspace, the ability to modify either flight path may be advantageous when rerouting one of the aircraft creates a secondary conflict and changing the other does not. The trial planner rejects resolutions that result in conflict with either another aircraft or CWAM polygon. There are conflicts the resolution algorithm could not solve. Other issues leading to the lower success rate as weather increases include the fact that the full set of heuristic maneuvers available for resolving conflicts is not implemented for resolving weather conflicts. These maneuvers include speed and compound horizontal reroutes with temporary altitude changes. At the time of this writing, some of these maneuvers were ready for weather avoidance testing but still not available for this study.

Figures 10 and 11 show original and trial flight plans generated by the iterative resolution process described in Fig. 1 for achieving a resolution that is free of traffic and weather conflicts. The original flight trajectory is shown in Fig. 10. The vertex and endpoint of the trajectory line represent the two downstream waypoints. Multiple CWAM polygons and other traffic are depicted in the vicinity. The other aircraft show are not necessarily all the aircraft that could cause a secondary conflict. The resolution algorithm will select maneuvers from a list based on the conflict geometry. The list is based on heuristics that mimic current pilot-controller operational practices. The list is also dependent

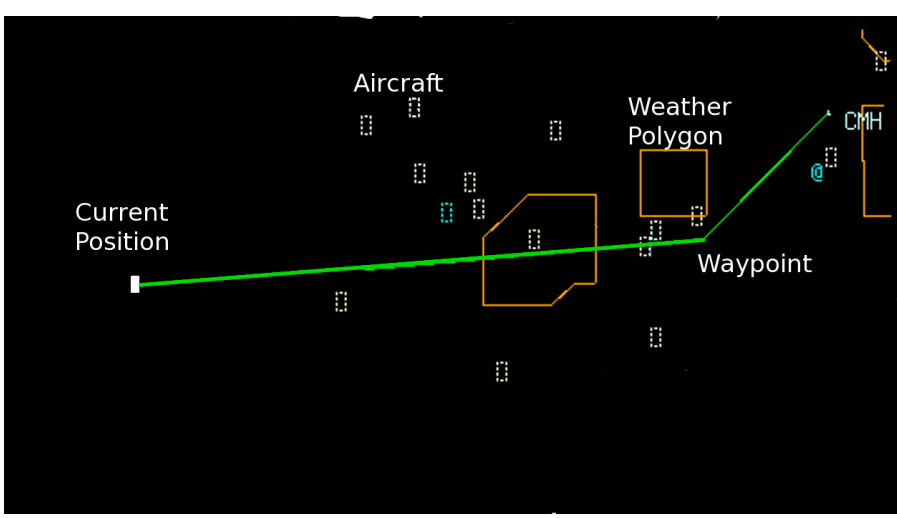

Figure 10. Original flight plan is in conflict with two weather polygons and other aircraft (square symbols). on aircraft type and performance. For weather encounters, CTAS creates a head on conflict geometry at the location where the aircraft trajectory intercepts the weather polygon. The resolution algorithm generates and checks maneuvers to avoid the conflict until it is resolved, or all the maneuvers are attempted, or the process runs out of time. CTAS is designed around a real-time architecture and each process has a defined amount of time to complete its task. Figure 11 shows the trial flight plan trajectories. The algorithm generates small angle deviations before larger angles. In general, one vertex maneuvers are attempted first, followed by two vertex maneuvers, then temporary altitude changes (altitude changes did not work in the present study). One vertex maneuvers have one auxiliary waypoint before rejoining the original flight plan whereas two vertex maneuvers have two auxiliary waypoints. The two vertex maneuvers result in a path parallel and offset to the original flight plan and bypass the first downstream waypoint and rejoin the flight plan at the second waypoint. In this case, no maneuver generated and checked for additional conflicts was able to resolve the conflict.

Depending on the size and location of the weather polygons and the number of other aircraft in the vicinity, there may not be a maneuver that can resolve the weather conflict using the heuristics normally employed to resolve aircraft-to-aircraft conflicts. The resolution algorithm was designed to avoid other aircraft that are many times smaller in size than the weather polygons investigated in this study. Information about the size of the weather polygons is not transferred to the algorithm. Additionally, the algorithm has limitations in the size of the rerouting, mainly that the total reroute should be less than 5 minutes of additional flying time and the maximum distance between auxiliary waypoints is 80 nautical miles. A parametric study of these a priori defined options might

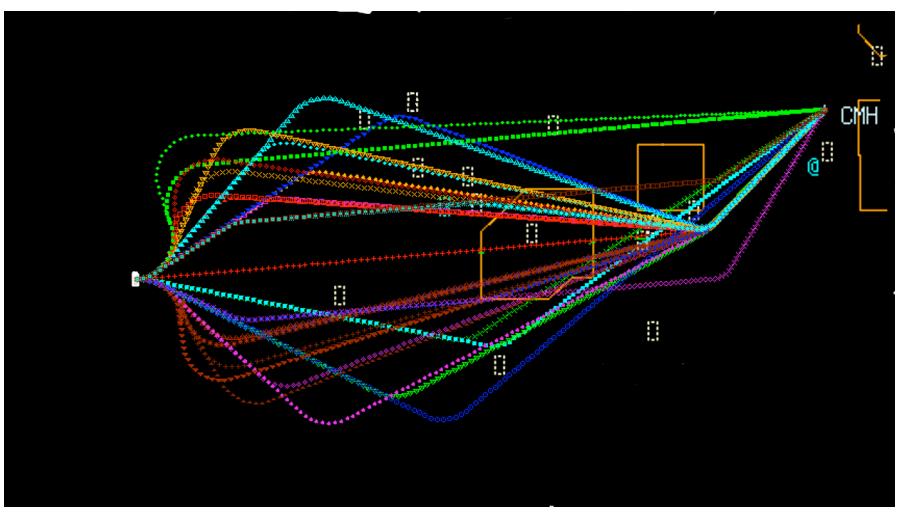

Figure 11. Original and proposed 4-D trajectories that did not resolve a conflict with the two weather polygons and other aircraft. 
find better combinations that resolve more conflicts but care would have to be taken to ensure the resulting maneuvers continue to be representative of current practice and their operational acceptance would remain high.

\section{B. Distribution of Maneuvers}

It was found that use of the automatic resolution logic for resolving conflicts between traffic and weather sometimes resulted in aircraft being rerouted under the weather constraint. This is clearly an unacceptable weather avoidance maneuver and future implementations must ensure that weather avoidance logic does not attempt to route aircraft under weather cells. Out of a total of 2774 weather avoidance maneuvers in this analysis, 149 "successful" resolutions were found to have been routed under weather and were removed from the results. These resolutions were removed from all results.

Figure 12 shows the distribution of successful horizontal reroute maneuvers generated by the resolution algorithm for both traffic and weather conflicts. The lines between the symbols are not intended to show a trend but are there only to aid the reader in seeing the general trends as the one-degree angle increment makes the plot cluttered, otherwise. Table 1 shows there were a total of 606 conflicts in this scenario but the total number of maneuvers attempted to resolve the conflict exceeded 84,000. Figure 12 represents 444 successful resolutions. This

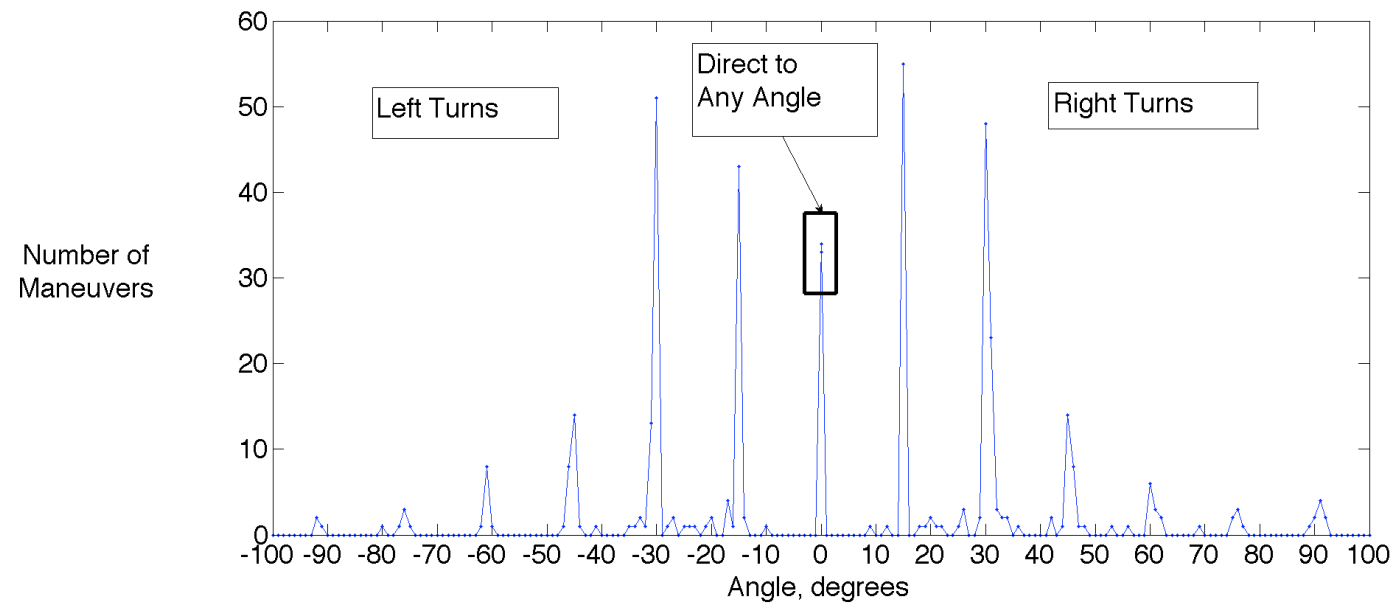

Figure 12. Number of successful left turn, right turn, and direct to waypoint maneuvers that resolved conflicts for the medium traffic, bad weather scenario.

high total was accounted for by realizing that for each time cycle where an aircraft or weather conflict was not resolved, the conflicts stays in the queue until it is either resolved or the time to conflict is less than the values in Fig. 1. At the next time cycle, another attempt is made to resolve the conflict with new information. It is noted that the resolution algorithm does attempt to use turn maneuvers greater than 90 degrees, however, most of the turning solutions are less than 50 degrees. The maneuvers direct to a downstream waypoint of any turning angle are tallied in the center of the plot at the zero degrees point. These maneuvers represent a reduction in the length of the flight versus the planned route. Whether the maneuvers represent a time savings is dependent on the wind. The difference in left versus right direct maneuvers is solely a function of the original flight plan and the conflict geometry. 


\section{Impact on Flights}

Table 2 shows the time savings resulting from resolution maneuvers direct to a downstream waypoint. The

\begin{tabular}{|c|c|c|c|c|}
\hline $\begin{array}{l}\text { Direct to a } \\
\text { Downstream } \\
\text { Waypoint }\end{array}$ & $\begin{array}{l}\text { Weather } \\
\text { Scenario } \rightarrow\end{array}$ & $\begin{array}{l}\text { Time savings } \\
\text { No Weather }\end{array}$ & $\begin{array}{c}\text { Time savings } \\
\text { Moderate } \\
\text { Weather }\end{array}$ & $\begin{array}{l}\text { Time savings } \\
\text { Bad Weather }\end{array}$ \\
\hline \multirow[t]{3}{*}{$\begin{array}{l}\text { Medium } \\
\text { Traffic }\end{array}$} & $\begin{array}{l}\text { Maximum } \\
\text { Mean } \\
\text { Median } \\
\text { Minimum } \\
\text { Std Deviation }\end{array}$ & $\begin{array}{l}828 \mathrm{~s} \\
62 \mathrm{~s} \\
28 \mathrm{~s} \\
3 \mathrm{~s} \\
\pm 29 \mathrm{~s}\end{array}$ & $\begin{array}{c}828 \mathrm{~s} \\
75 \mathrm{~s} \\
37 \mathrm{~s} \\
3 \mathrm{~s} \\
\pm 129 \mathrm{~s} \\
\end{array}$ & $\begin{array}{c}1315 \mathrm{~s} \\
109 \mathrm{~s} \\
47 \mathrm{~s} \\
3 \mathrm{~s} \\
\pm 238 \mathrm{~s} \\
\end{array}$ \\
\hline & Total Savings & $45 \mathrm{~m}$ & $59 \mathrm{~m}$ & $121 \mathrm{~m}$ \\
\hline & Total Count & 44 flights & 47 flights & 67 flights \\
\hline \multirow[t]{3}{*}{$\begin{array}{l}\text { High } \\
\text { Traffic }\end{array}$} & $\begin{array}{l}\text { Maximum } \\
\text { Mean } \\
\text { Median } \\
\text { Minimum } \\
\text { Std Deviation }\end{array}$ & $\begin{array}{c}237 \mathrm{~s} \\
45 \mathrm{~s} \\
35 \mathrm{~s} \\
0 \mathrm{~s} \\
\pm 48 \mathrm{~s} \\
\end{array}$ & $\begin{array}{c}247 \mathrm{~s} \\
65 \mathrm{~s} \\
31 \mathrm{~s} \\
3 \mathrm{~s} \\
\pm 67 \mathrm{~s} \\
\end{array}$ & $\begin{array}{c}261 \mathrm{~s} \\
49 \mathrm{~s} \\
30 \mathrm{~s} \\
0 \mathrm{~s} \\
\pm 54 \mathrm{~s} \\
\end{array}$ \\
\hline & Total Savings & $44 \mathrm{~m}$ & $42 \mathrm{~m}$ & $59 \mathrm{~m}$ \\
\hline & Total Count & 57 flights & 39 flights & 72 flights \\
\hline
\end{tabular}

resolution algorithm always tests maneuver direct to a waypoint first in its hierarchy. Table 2 shows a trend of maneuvers direct to downstream waypoint increasing time savings with increasing weather severity. This simulation did not proactively search for direct maneuvers, it only searched when there was a conflict. All the values shown in Table 2 saved time compared to the original route. Because wind data was not included in the simulation, no direct routing to a downstream waypoint resulted in a delay.

Large time savings are attributed to aircraft filing a flight plan that allowed for short-cuts. However, these short cuts did not take into account a wind field that may not have resulted in such large savings. This is supported in the medium traffic scenarios by the 828 and 1315 -second savings, 14 and 22 minutes, respectively. These flight plan routes were generated while considering the weather and winds of Dec. 21, 2007. The original and amended trajectories for both of these cases were investigated and it was noted that the original trajectory was significantly longer. For the medium traffic scenario, the large 30 second time saving differences between the mean and median values gives an indication of how much the mean was skewed toward the extreme values. There was also a corresponding increase in the standard deviation for the medium traffic scenarios.

The high traffic scenario mean time savings were less than the medium scenario, suggesting that the weather limited the unobstructed distance to downstream waypoint. The standard deviation is smaller than the medium traffic and the total savings is less than the medium scenario.

The simulated scenarios investigated do not account for traffic flow management initiatives that may have been in effect the day the flight plans were filed. Therefore, an increasing number of conflicts will lead to an increasing possibility of finding a direct to maneuver with significant savings. The strategy of first looking for a direct maneuver helps balance the total aggregate delay in these situations. In two out of the three high traffic scenarios, the total number of aircraft benefiting from a direct maneuver increased. The maximum savings for the high traffic cases were approximately equal and the standard deviation was much smaller for the moderate and bad weather scenarios. The average savings ranged from 45 to 109 seconds, though some flights had little or no reduction in flying time.

Table 3 shows the delays incurred as a result of all maneuvers other than direct-to resolutions. As expected, aggregate delays increased with the severity of the weather. This delay increase ranged from 5 to 12 times when compared to the no weather baseline although the mean delay per flight appears evenly distributed, remaining under 81 seconds. This shows that as more flights were affected, they all experienced a similar amount of delay. It was 
also noted that some flights experienced a time savings even though the assigned maneuver was not directly to a downstream waypoint. This occurs when a flight is given an auxiliary waypoint before rejoining the flight plan

Table 3. Analysis of delay incurred by rerouting of aircraft

\begin{tabular}{llccc}
\hline \hline & Weather & No Weather & $\begin{array}{c}\text { Moderate } \\
\text { Weather }\end{array}$ & Bad Weather \\
\hline \hline \multirow{4}{*}{ Sedium } & Minimum & $11 \mathrm{~s}$ & $-50 \mathrm{~s}$ & $-114 \mathrm{~s}$ \\
Traffic & Mean & $48 \mathrm{~s}$ & $70 \mathrm{~s}$ & $81 \mathrm{~s}$ \\
& Median & $39 \mathrm{~s}$ & $40 \mathrm{~s}$ & $56 \mathrm{~s}$ \\
& Maximum & $159 \mathrm{~s}$ & $281 \mathrm{~s}$ & $326 \mathrm{~s}$ \\
& Std Deviation & $\pm 29 \mathrm{~s}$ & $\pm 50 \mathrm{~s}$ & $\pm 56 \mathrm{~s}$ \\
\cline { 2 - 5 } & Total Delay & $41 \mathrm{~m}$ & $277 \mathrm{~m}$ & $506 \mathrm{~m}$ \\
\cline { 2 - 5 } & Total Count & $52 \mathrm{flights}$ & $235 \mathrm{flights}$ & $377 \mathrm{flights}$ \\
\hline \hline \multirow{4}{*}{ High } & Minimum & $-233 \mathrm{~s}$ & $-6 \mathrm{~s}$ & $-203 \mathrm{~s}$ \\
& Mean & $45 \mathrm{~s}$ & $67 \mathrm{~s}$ & $69 \mathrm{~s}$ \\
& Median & $39 \mathrm{~s}$ & $40 \mathrm{~s}$ & $40 \mathrm{~s}$ \\
& Maximum & $249 \mathrm{~s}$ & $268 \mathrm{~s}$ & $318 \mathrm{~s}$ \\
& Std Deviation & $\pm 48 \mathrm{~s}$ & $\pm 44 \mathrm{~s}$ & $\pm 52 \mathrm{~s}$ \\
\cline { 2 - 5 } & Total Delay & $122 \mathrm{~m}$ & $398 \mathrm{~m}$ & $714 \mathrm{~m}$ \\
\cline { 2 - 5 } & Total Count & $162 \mathrm{flights}$ & $358 \mathrm{flights}$ & $617 \mathrm{flights}$ \\
\hline \hline
\end{tabular}

route but the total distance is still shorter than the original flight plan. The smaller standard deviations, as compared to Table 2, shows the data are not spread out over the range. The difference in maximum delay amongst the rerouted flights is under five minutes.

\section{Rerouting Effects on TMA Schedule}

With the weather translation model integrated into CTAS, it is now possible to show the impact the rerouting has on TMA schedules. Since TMA is a tool within CTAS, it uses the same trajectory predictor. Therefore, changes in an aircraft trajectory can be reflected as a change in schedule in the TMA timeline. Figure 13 shows a typical weather conflict avoidance maneuver that is conflict free of weather and aircraft up to 20 minutes from the current position of the aircraft. Figure 12 shows the original flight path trajectory of the SKW2605 aircraft, depicted as a green line. The curved white lines show the TMA meter fix arcs and the white dots are numerous arrival fixes of the destination airport. All other traffic has been filtered out. The aircraft is flying north to south and originates in the Chicago ARTCC (ZAU) en route to a meter fix. High altitude airspace of ZID is shown in the center of the figure with brown outlines. The flight data block of the aircraft is shown with altitude, ground speed, call sign, and aircraft type. Altitude is in hundreds of feet and ground speed is in knots. The smaller polygons in Fig. 13 are CWAM polygons with an 80 percent probability of deviation.

Figure 14 shows the successful conflict resolution trajectory path to avoid the CWAM polygon. The new trajectory is clear of the weather constraints and other aircraft in the center airspace and the original meter fix still in the flight plan. 


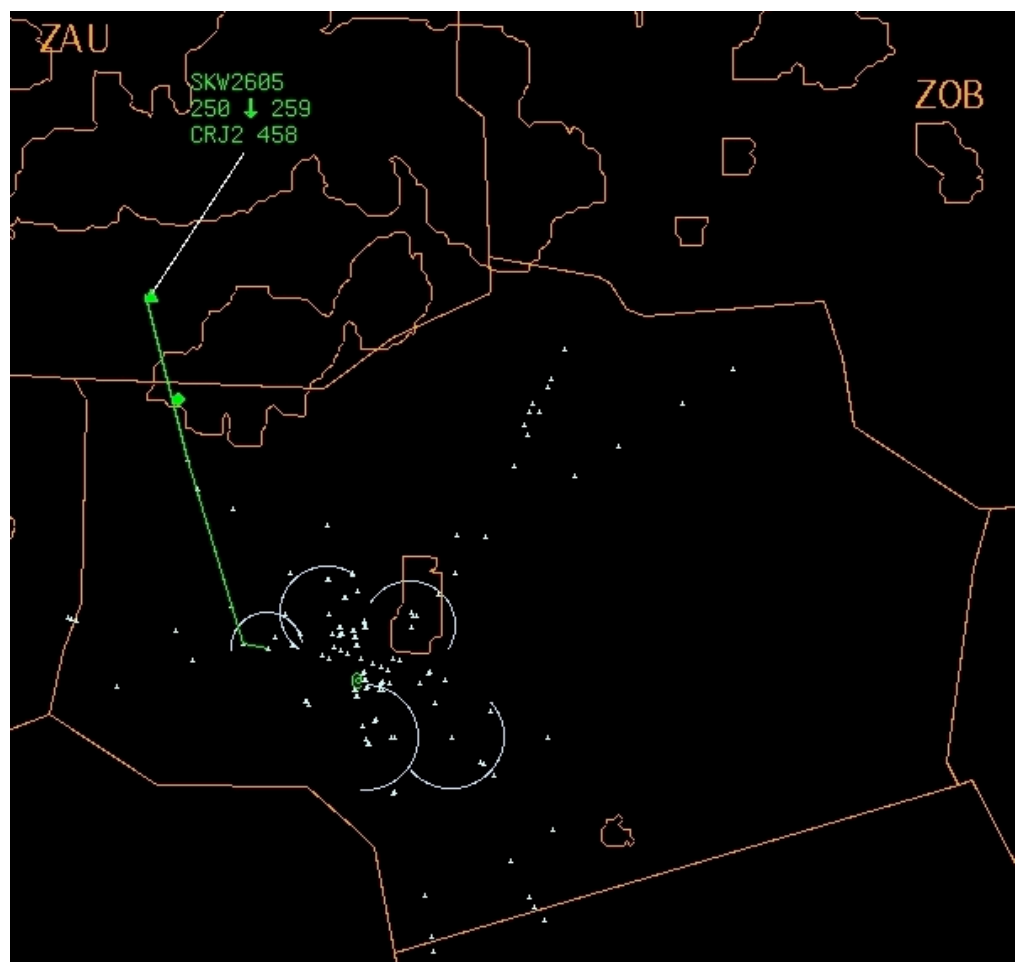

Figure 13. Aircraft conflict with 80 percent CWAM contour automatically detected.

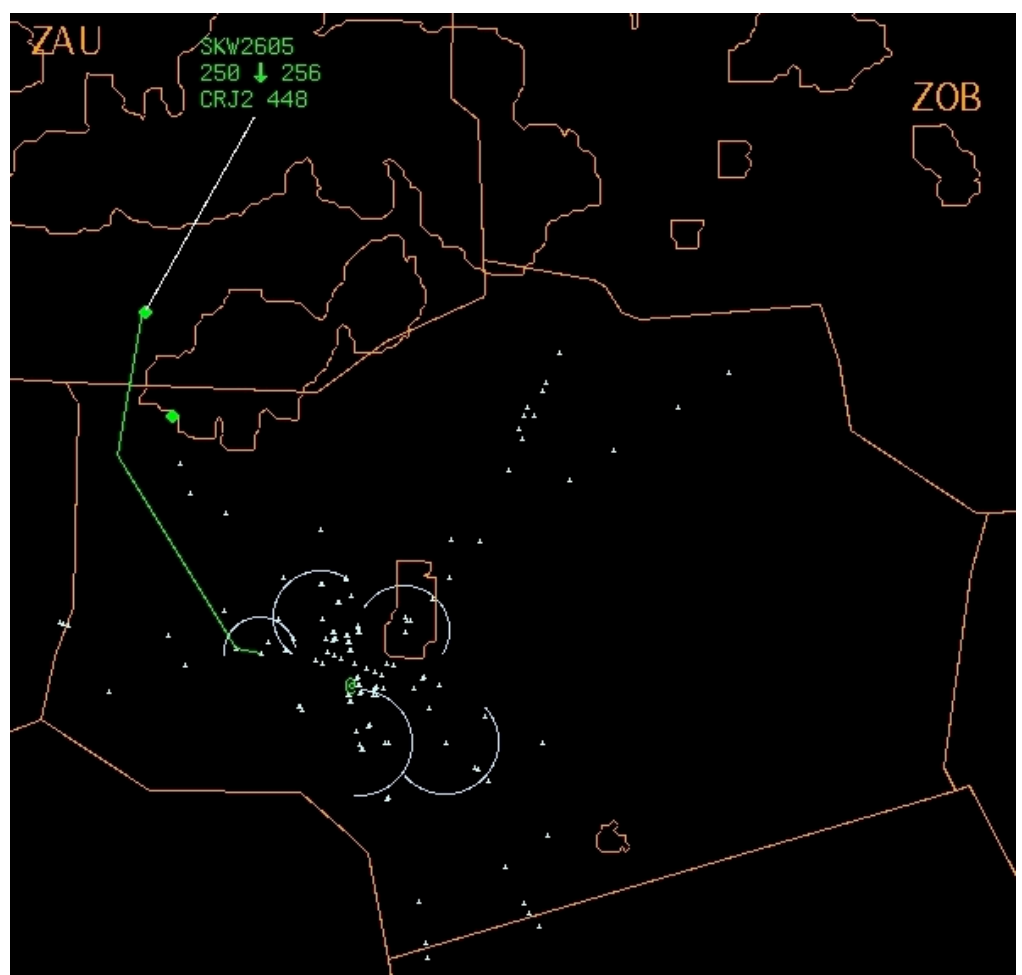

Figure 14. Aircraft automatically rerouted to avoid the 80 percent CWAM contour. 


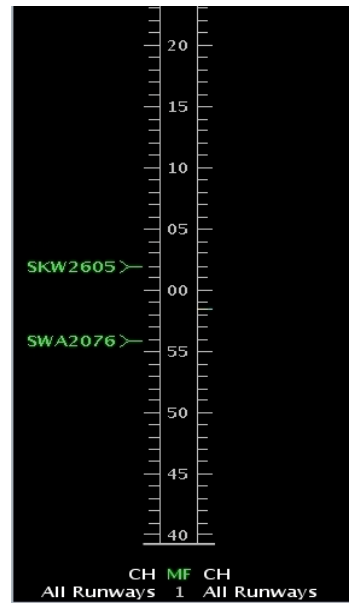

Figure 15. TMA timeline before rerouting

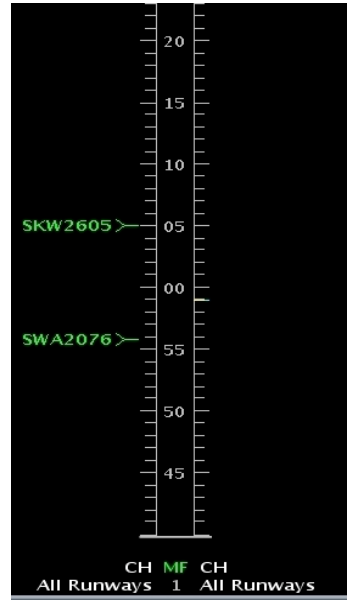

Figure 16. TMA timeline after rerouting

Figures 15 and 16 show the effect of the aircraft reroute around the weather using the TMA module in CTAS. The bottom of the figure represents the current time in minutes after the hour at the meter fix and the aircraft and numbers move toward the bottom as if on a ticker tape. When an aircraft is rerouted by a flight plan amendment, all modules in CTAS are able to take advantage of the updated information. The SKW2605 flight shown in Fig 15 is scheduled to arrive at the meter fix at 2 minutes after the hour. Figure 16 shows flight after rerouting around weather. The aircraft is scheduled to arrive at the meter fix at 5 minutes after the hour. The delay for this rerouting is about 3 minutes.

\section{Conclusions}

An automatic conflict resolution algorithm in the Center-TRACON Automation System for en route airspace was extended to resolve traffic and convective weather conflicts in an integrated detection and resolution methodology. This study evaluated the extension of an algorithm to automatically resolve aircraft to aircraft conflicts already in the Center-TRACON Automation System to also avoid weather conflicts ${ }^{13}$. The approach described in this paper was taken for the following reason. Using a separate weather avoidance algorithm would require that it work with the current aircraft conflict resolution algorithm in the Center-TRACON Automation System or an entirely new algorithm that resolved both weather and aircraft conflicts would need to be created.

Aircraft trajectories that were predicted to fly into weather constrained areas as defined by the Convective Weather Avoidance Model were analyzed to find conflict free resolution trajectories in the same manner as other aircraft-to-aircraft conflicts. The simulation combined two clear weather traffic scenarios with three weather scenarios that increased from no weather to significant weather.

The success rate for resolving aircraft-to-aircraft conflicts without weather constraints is 96 percent or greater. The four percent that were unresolved in the high traffic no weather scenario had secondary conflicts. Success rate for high traffic with moderate weather was 91 percent while the high traffic with bad weather success rate dropped to 72 percent. The success rate decreases with increasing convective weather because there may not be a maneuver that can resolve the weather conflict using the heuristics normally employed to resolve aircraft-to-aircraft conflicts. The success depends on the size and location of individual weather polygons, total area coverage, and the number of other aircraft in the vicinity. Comparing the total number of conflicts to the no weather scenario, the bad weather scenarios had six times and four times as many conflicts for the medium and high traffic simulations, respectively.

Individual and aggregate delays due to rerouting were analyzed. The aggregate delay almost tripled from 41 minutes to 122 minutes due to increasing traffic from the medium to the high scenarios without weather but the mean delay was 48 seconds or less. The bad weather scenario increased the aggregate delay to 506 and 714 minutes for the two traffic scenarios. The mean delay remained under 90 seconds in all scenarios for individual flights affected and showed the rerouted flights experienced a similar delay as the number of flights affected increased. Additionally, other flights experienced a mean savings ranging between 45-109 seconds because rerouting shortened the flight path. Direct maneuvers always resulted in a savings because the simulation did not include wind.

Changes to the Traffic Management Advisor schedule resulting from automatically rerouting aircraft to avoid both aircraft and weather was shown on the TMA timeline. The Traffic Management Advisor uses the same 4-D 
trajectory predictor as other elements in CTAS. Therefore, software changes to allow visualization of aircraft flight times on the TMA timeline were minimal as time steps are always computed and available.

This study was not intended to be a final solution in automatic resolutions of aircraft and weather conflicts but the success rate shows the method has merit. Experience has been gained in integrating and utilizing weather translation models in the Center-TRACON Automation System decision support tool. Implementing the full complement of resolution maneuvers should improve the resolution algorithm's performance.

\section{Future Work}

Implementing temporary altitude, speed, and compound maneuvers will be the next step in improving the results presented in this paper. Compound maneuvers consist of horizontal reroutes combined with temporary altitude changes.

A future enhancement to the resolution algorithm could be to evaluate the size of gaps between weather constraints to evaluate if those gaps are likely to be traversed by a flight. Closing off gaps that flights are unlikely to traverse should improve system robustness by decreasing the number of rejected maneuvers.

Another option to be considered when a conflict cannot be resolved is constraint relaxation. This involved increasing the allowable CWAM probability, i.e. from 80 percent to 90 percent, and minimizing the dwell time in the CWAM polygon. If the conflict is successfully resolved with either option, the effect of the reroute on the aircraft's schedule should be analyzed.

If the improvements discussed above do not achieve the required success rates for an automated system, integrating a separate weather avoidance that include a secondary aircraft conflict detection and resolution algorithm may be required.

\section{References}

${ }^{1}$ Hoang, T., and Swenson, H., "The Challenges of Field Testing the Traffic Management Advisor (TMA) in an Operational Air Traffic Control Facility," NASA TM-112211, Aug. 1997.

${ }^{2}$ McNally, B. D., Engelland, S., Bach, R., Chan, W., Brasil, C., Gong, C., Frey, J., Vincent, D., "Operational Evaluation of the Direct-To Controller Tool," 4th USA/Europe Air Traffic Management R\&D Seminar, Santa Fe, NM, USA, Dec. 3-7, 2001.

${ }^{3}$ FAA REDAC, 2007, "Weather-Air Traffic Management Integration Final Report," Weather -ATM Integration Working Group (WAIWG) of the National Airspace System Operations Subcommittee, Federal Aviation Administration (FAA) Research, Engineering and Development Advisory Committee (REDAC), Oct. 3, 2007.

${ }^{4}$ Erzberger, H. (2006), "Automated Conflict Resolution for Air Traffic Control," 25th International Congress of the Aeronautical Sciences (ICAS), Hamburg, Germany, Sep. 3-7, 2006.

${ }^{5}$ Windhorst, R. D. and Refai, M., "Convective Weather Avoidance with Uncertain Weather Forecasts", IEEE/AIAA 28 ${ }^{\text {th }}$ Digital Avionics Systems Conference, IEEE, 2009.

${ }^{6}$ Chan, William, Mohamad Refai, Rich DeLaura: "Validation of a Model to Predict Pilot Penetrations of Convective Weather", 7th AIAA Conference on Aviation, Technology, Integration and Operations, Belfast, Ireland, Sep. 2007.

${ }^{7}$ Hoang, T., and Swenson, H., "The Challenges of Field Testing the Traffic Management Advisor (TMA) in an Operational Air Traffic Control Facility," NASA TM-112211, Aug. 1997.

${ }^{8}$ DeLaura, R., and Evans, J., “An Exploratory Study of Modeling En Route Pilot Convective Storm Flight Deviation Behavior," Proceedings of the $12^{\text {th }}$ Conference on Aviation, Range, and Aerospace Meteorology, Atlanta, 2006.

${ }^{9}$ Klingle-Wilson, D., J. Evans: "Description of the Corridor Integrated Weather System (CIWS) Weather Products," MIT Lincoln Laboratory Project Report ATC-317, Aug. 1, 2005.

${ }^{10}$ Grabbe, S, Sridhar, B., Mukherjee, A., "Integrated Traffic Flow Management Decision Making," AIAA Meeting Papers on Disc, Vol. 14 No. 9 (GNC/AFM/MST), AIAA, Reston, VA, 2009.

${ }^{11}$ Erzberger, H., "Automated Conflict Resolution for Air Traffic Control," $25^{\text {th }}$ International Congress of the Aeronautical Sciences (ICAS), Hamburg, Germany, Sep. 3-7, 2006.

12 Anon., "NEXRAD National Reflectivity Mosaics," [Online database] URL http://www4.ncdc.noaa.gov/cgiwin/wwcgi.dll?wwNexrad Images2, National Climatic Data Center, Ashville, NC, 2009.

${ }^{13}$ McNally, D., and Thipphavong, D., "Automated Separation Assurance in the Presence of Uncertainty," International Council for the Aeronautical Sciences (ICAS) 2008 Congress, Anchorage, AK, Sep. 14-19, 2008. 